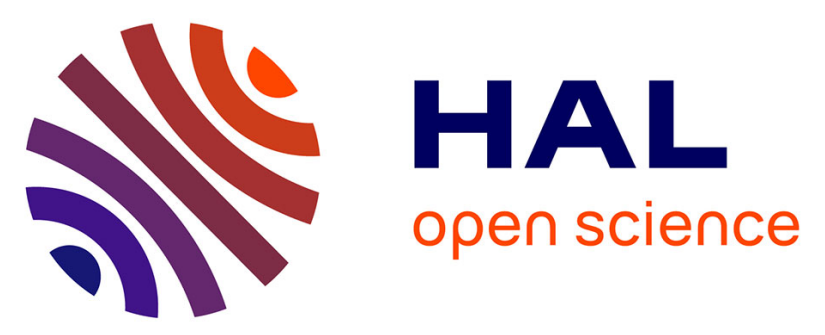

\title{
Nasal cavity shape in unilateral choanal atresia and the role of fetal ventilation in facial growth
}

\author{
S. Ferrier, Q. Hennocq, N. Leboulanger, V. Couloigner, F. Denoyelle, Y.
} Heuzé, R.H. H Khonsari

\section{> To cite this version:}

S. Ferrier, Q. Hennocq, N. Leboulanger, V. Couloigner, F. Denoyelle, et al.. Nasal cavity shape in unilateral choanal atresia and the role of fetal ventilation in facial growth. Journal of Stomatology, Oral and Maxillofacial Surgery, 2020, 10.1016/j.jormas.2020.05.021 . hal-02985636

\section{HAL Id: hal-02985636 \\ https://hal.science/hal-02985636}

Submitted on 3 Dec 2020

HAL is a multi-disciplinary open access archive for the deposit and dissemination of scientific research documents, whether they are published or not. The documents may come from teaching and research institutions in France or abroad, or from public or private research centers.
L'archive ouverte pluridisciplinaire HAL, est destinée au dépôt et à la diffusion de documents scientifiques de niveau recherche, publiés ou non, émanant des établissements d'enseignement et de recherche français ou étrangers, des laboratoires publics ou privés. 
Original article

\title{
Nasal cavity shape in unilateral choanal atresia and the role of fetal ventilation in facial growth
}

\author{
S. Ferrier ${ }^{\mathrm{a}, *}$, Q. Hennocq ${ }^{\mathrm{b}}$, N. Leboulanger ${ }^{\mathrm{a}}$, V. Couloigner ${ }^{\mathrm{a}}$, F. Denoyelle ${ }^{\mathrm{a}}$, Y. Heuzé $^{\mathrm{c}}$, \\ R.H. Khonsari ${ }^{\text {b }}$ \\ ${ }^{a}$ Service d'otorhinolaryngologie et chirurgie cervico-faciale, Hôpital Universitaire Necker-Enfants Malades, Assistance Publique-Hôpitaux de Paris, \\ Centre de Référence des Malformations ORL rares MALO, Filière Maladies Rares TeteCou, Université Paris Descartes, Université Sorbonne Paris Cité, \\ 149, rue de Sèvres, 75015 Paris, France \\ ${ }^{\mathrm{b}}$ Service de chirurgie maxillo-faciale et chirurgie plastique, Hôpital Universitaire Necker-Enfants Malades, Assistance Publique-Hôpitaux de Paris, \\ Centre de Référence des Fentes et malformations faciales MAFACE, Filière Maladies Rares TeteCou, Université de Paris, Paris, France \\ ${ }^{\mathrm{C}}$ CNRS, University Bordeaux, MC, PACEA - De la Préhistoire à l'Actuel : Culture, Environnement et Anthropologie, UMR5199, Pessac, France
}

\section{A R T I C L E I N F O}

\section{Article history:}

Received 3 May 2020

Accepted 19 May 2020

\section{Keywords:}

Choanal atresia

Geometric morphometrics

Craniofacial growth

Functional matrix

3D imaging

\begin{abstract}
A B S T R A C T
Objective: The respiratory movements of fetal amniotic fluid areconsidered by certains cleft surgery teams to contribute to the growth of the nasal cavities (NC). To assess this functional hypothesis, we considered a group of patients with unilateral choanal atresia (CA) as a model of unilateral absence of amniotic fluid flux in the NC, and compared their NCs shape to age-matched controls.

Material and methods: Three-dimensional reconstructions of NC were performed using Avizo 9.7 (Thermo Fisher Scientific, MA, USA), based on CT-scans of 32 patients with unilateral CA and 96 age- and gender-matched controls. Landmarks were placed on anatomical structures of NC. Procrustes superimpositions and principal component analysis were performed. Anatomically relevant Euclidean distances were computed using the coordinates of selected landmarks - maxillary length, piriform orifice width, choanal width - and tested using multivariate analysis. Growth rates between patients and controls for these distances were screened for correlations.

Results: The atretic NC was significantly deformed when compared to the control cases: Procrustes distance was $0.28(P<0.0001)$. The maxillary length and width of the atretic choana were significantly decreased compared to controls $(-2.95 \mathrm{~mm}$ and $-1.35 \mathrm{~mm}$ respectively, $P<0.001)$. There were no differences in growth rates between CA and controls, except for the choanal width on the atretic side. Conclusion: NCs in CA were significantly different from controls. More precisely, the maxillary length was significantly reduced in the CA group. There was no other major shape difference between the NC in CA and controls. NC seems to develop despite abnormal fetal ventilation.
\end{abstract}

(c) 2020 Published by Elsevier Masson SAS.

\section{Introduction}

\subsection{Choanal atresia and nasal cavity formation}

Choanal atresia (CA) is a complete congenital absence of communication between the posterior nasal cavity (NC) and the nasopharynx. Choanal stenosis is a narrowing of the posterior choana without complete obstruction. In clinical practice, both anomalies are referred to as CA. The incidence of CA varies between $1 / 5000$ and $1 / 9000$ births. CA is a characterized condition since the

\footnotetext{
* Corresponding author.

E-mail address: suzanne.ferrier@aphp.fr (S. Ferrier).
}

end of the 18th century and was initially described by Johann Roederer in 1755; Carl Emmert reported the first surgical repair of CA in 1851, based on the perforation of the atretic plate [1].

CA has no sexual predominance but right-sided CA may be more prevalent, even though this point is not consensual [2-4]. Three types of CA are reported in the literature:

- membranous CA;

- bony CA;

- combined forms (membranous + bony).

In the beginning of the 20th century, it was believed that $90 \%$ of CA were bony and that $10 \%$ were membranous[2-4]. Nevertheless, with the development of diagnoses based on CT-scans, more recent 
studies report $70 \%$ of combined CA vs. $30 \%$ of bony CA [2-4]. Unilateral CAs are more frequently isolated than bilateral CAs, which can be associated with a wide range of syndromes - mostly CHARGE association and syndromic craniosynostosis syndromes such as Crouzon, but also less common conditions such as Pfeiffer, Antley-Bixler, Marshall-Smith, Schinzel-Giedon and Treacher Collins syndromes [4-6]. Interestingly, craniosynostoses are associated with choanal stenosis rather than with atresia [6]. As a whole, about $51 \%$ of CAs are associated with other malformations.

Four diversely convincing theories have been proposed in the literature to account for the occurrence of CA, without much support from developmental biology $[7,8]$ :

- Persistence of the bucco-nasal membrane of Hochstetter, formed by the olfactive plate - inside the nasal pit mesoderm - and the oral cavity epithelium [9]; the normal dehiscence of this membrane results in the formation of the primary choana;

- Persistence of the bucco-pharyngeal membrane, which is the superior limit of the primary gut, spontaneously rupturing in normal conditions between the 3rd and the 4th week of gestation [10];

- Adherences formed by an abnormal persistence of mesodermal tissue [8];

- Migration anomalies of neural crest cells [8].

Toxic factors such as thioamides have also been implicated in the origin of CA [2-4,7]. In fact, CA is generally considered as a component of carbimazole embryopathy: for instance, in a study reporting 20 newborns exposed to carbimazole, 11 presented with CA [11]. Furthermore, increased uni- and/or bilateral CA have been reported in embryos exposed to methymazole between the 3rd and 7 th weeks of embryologic development $[12,13]$.

Several genes are involved in CA, such as Raldh3 in mice and CHD7 in humans. Inactivation of Raldh3 causes bilateral CA in mouse models; interestingly, the Raldh3 protein is expressed in the posterior and central mesoderm of the nasal cavity and nostrils and is part of the retinoic acid signaling pathway. Raldh3 knock-out mice did not develop CA when mothers were treated with nonteratogenic doses of retinoic acid [14,15]. Seven to $29 \%$ of CA cases reported in the literature are part of a CHARGE association, and 35 to $65 \%$ CHARGE association cases have CA. The most frequent genetic anomaly in CHARGE association is the CHD7 mutation, and this gene is currently the only human candidate for causing CA [16]. The mechanism linking CA and CHD7 mutations is not currently understood.

\subsection{Nasal cavity growth}

Beyond morphogenesis issues, several authors have investigated the role of external mechanical factors on the growth of the nasal cavities. For instance, the flux of amniotic fluid during fetal respiration has been proposed to be a major contributor to prenatal maxillary growth [17]. In patients with cleft lip and palate, different pressures in the nasal cavities on both sides of the septum, during fetal amniotic respiration, have been incriminated in the deformation of the septum and alae nasi [18]. In this context, $\mathrm{CA}$ is an interesting model to assess these mechanical theories: CA should theoretically induce a collapse of the nasal cavity, as the atretic side is not subjected to amniotic liquid dynamics in atresia, and to abnormal flux in stenosis.

The reference for the diagnosis of CA is 2D CT-scan: atretic cavities have a narrower nasal floor and mid-orbital axis when compared to the contralateral side; the vomer is similarly wider on the atretic side $[19,20]$. Various methods have been described to quantify the shape of the nasal cavities, based on segmentation of CT-scan data and 3D landmarking [6], or on statistical shape modeling [21]. Here we assessed the shape of the nasal cavities in CA using 3D geometric morphometrics, and discussed our results in the light of the current theories relating external mechanical factors and centro-facial growth.

\section{Material and methods}

\subsection{Population}

We included all patients admitted for unilateral CA surgery between 2007 and 2018, with available good quality pre-operative CT-scans. Patient charts were retrospectively reviewed and the following clinical parameters were collected: sex, age at CTscan, CA side, type of CA, associated conditions or craniofacial malformations. Patients with craniofacial malformations were excluded (cleft lip and palate, Crouzon syndrome).

Age-matched normal CT-scans performed for minor trauma were used as controls. This study was assessed by the NeckerEnfants Malades Ethics Committee and complied with the local ethical regulations (CNEM 2020-12).

\subsection{Landmarking}

Landmarking was performed using Avizo 9.7 (Thermo Fisher Scientific, MA, USA) based on 3D reconstructions segmented based on a single threshold comprised between 140 and 290 Hounsfield units. Twenty bony landmarks were placed by a single observer (SF, Table 1, Fig. 1) and the 3D coordinates of the landmarks were exported. Left CA coordinates were transformed into right CAs in order to obtain a uniform CA group for Procrustes superimposition (see below).

We assessed intra- and inter-observer reproducibility based on a dataset of 10 normal CT-scans. Two operators (SF and RHK) landmarked these 10 scans three consecutive times. The concordance correlation coefficient (CCC) agreement of Lin [22] was computed for each operator and landmark. An overall concordance correlation coefficient (OCCC) was also computed [23].

\subsection{Morphometric analyses}

Procrustes superimposition based on the 3D coordinates of the 20 landmarks was performed using MorphoJ [24]. Left and right CA were grouped together after coordinate transformation of left forms into right forms.

Five Euclidean linear distances were computed:

- distances between landmarks 2-3 and 2-4 (respectively right and left piriform aperture width);

- distances between landmarks 12-13 and 12-14 (respectively left and right choanal width);

- distance between landmarks 2-12 (maxillary length).

\subsection{Statistical analysis}

Principal component analysis (PCA) based on the covariance matrix of the Procrustes shape coordinates was performed. The Procrustes distances between 'controls', 'right CA', 'left CA transformed into right $\mathrm{CA}^{\prime}$ ' and 'right $\mathrm{CA}+$ left $\mathrm{CA}$ transformed into right $\mathrm{CA}^{\prime}$ ' were computed. P-values were computed from permutation tests $(10,000$ permutation rounds) for Procrustes distances among groups. PCA defined a morphospace into which groups were separated by a distance referred to as Procrustes distance (d). For large enough Procrustes distances (determined by permutation tests), groups will be considered significantly different in shape. 
Table 1

Anatomical description of the landmarks used for modeling the nasal cavities. Mid.: midline; Bilat.: bilateral.

\begin{tabular}{|c|c|c|c|}
\hline Landmark & Structure & Name & Description \\
\hline 1 & Mid. & Nasion & Intersection of nasal and frontal bones \\
\hline 2 & Mid. & Anterior nasal spine & Triangular bony protrusion extending from the maxillary incisor crest \\
\hline 3 & Bilat. & Infero-lateral angle of right piriform aperture & Lateral angles of piriform apertures \\
\hline 4 & Bilat. & Infero-lateral angle of left piriform aperture & \\
\hline 5 & Bilat. & Right lachrymal duct, superior opening & Antero-superior angle of lachrymal ducts, superior opening \\
\hline 6 & Bilat. & Left lachrymal duct, superior opening & \\
\hline 7 & Bilat. & Right inferior turbinate head & Inferior turbinate, most anterior part, on its medial side \\
\hline 8 & Bilat. & Left inferior turbinate head & \\
\hline 9 & Bilat. & Right medium turbinate head & Medium turbinate, most anterior part, on its medial inferior angle \\
\hline 10 & Bilat. & Left medium turbinate head & \\
\hline 11 & Mid. & Vomer insertion on sphenoid bone & \\
\hline 12 & Mid. & Posterior nasal spine & Bony protrusion formed by posterior ends of internal sides of palatine plates. \\
\hline 13 & Bilat. & Postero-lateral angle of left palatine plate & On a posterior view, external angle of palatine plates, at the junction with the pterygoids \\
\hline 14 & Bilat. & Postero-lateral angle of right palatine plate & \\
\hline 15 & Bilat. & Superior angle of left choanal arch & At the center of choanal arch, superior concavity \\
\hline 16 & bilat. & Superior angle of right choanal arch & \\
\hline 17 & bilat. & Left inferior turbinate rear & Inferior turbinate, most posterior part, on its medial side \\
\hline 18 & Bilat. & Right inferior turbinate rear & \\
\hline 19 & Bilat. & Left medium turbinate rear & Medium turbinate, most posterior part, on its medial inferior angle \\
\hline 20 & Bilat. & Right medium turbinate rear & \\
\hline
\end{tabular}

A multivariate analysis based on a linear model adapted for each distance was used to compare the Euclidean distances between CA patients and controls. The analysis was adjusted on the following variables: distance value (in $\mathrm{mm}$ ), CA vs. control, and age. Growth rate correlations were estimated for each of these distances.

\section{Results}

Thirty-two children with CA were included into the study; sex ratio was 11 males for 21 females; age at CT was 1 day to 7 years of age ( $451.4 \pm 647.9$ days): $12 / 32$ patients were less than 3 months of age; 23/32 were less than 24 months of age, 3/32 were between
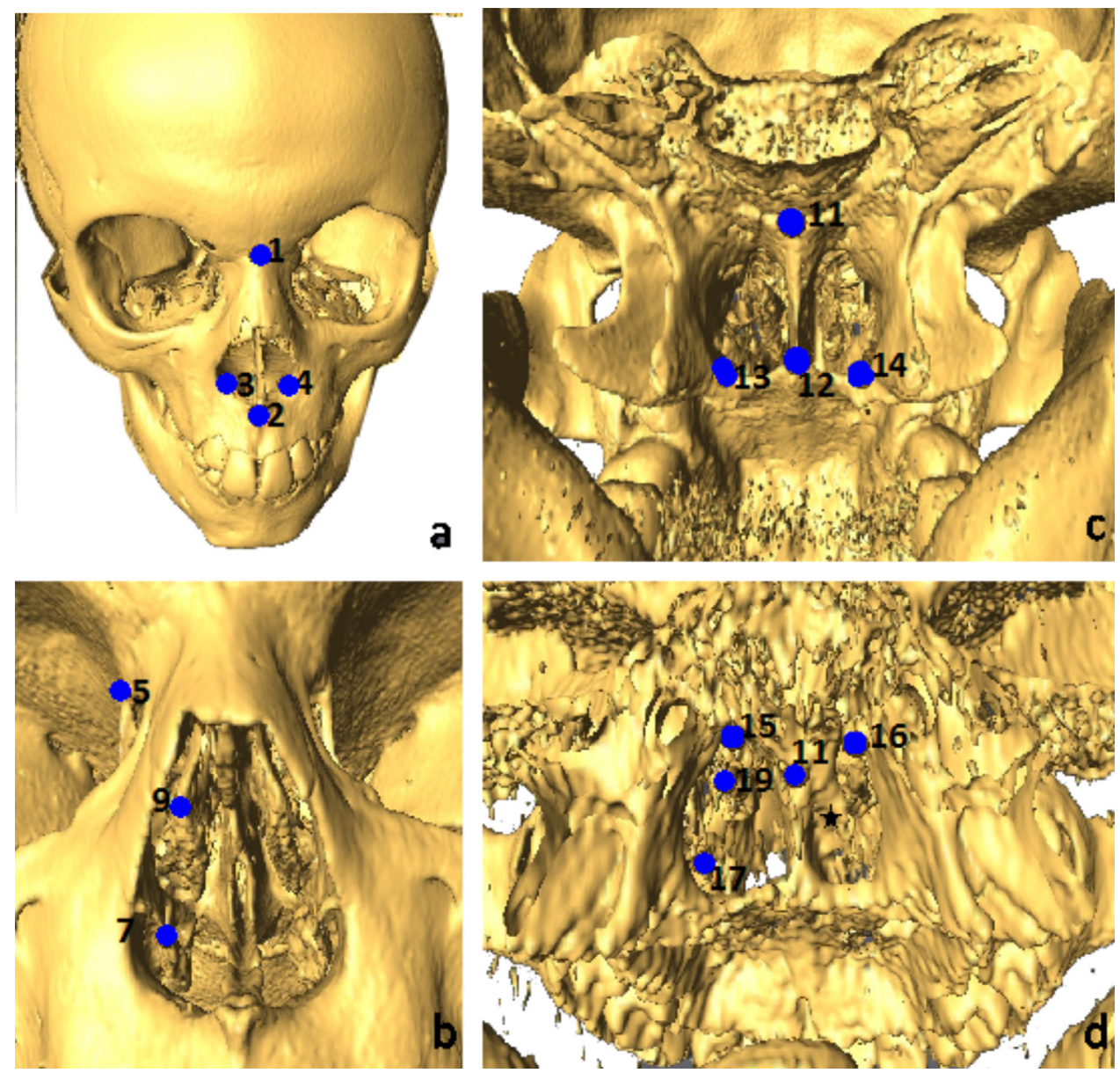

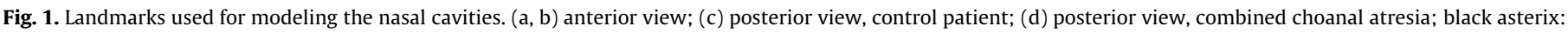
atretic plate. 
Table 2

Description of the population. Sd: standard deviation.

\begin{tabular}{|c|c|c|c|c|c|c|}
\hline & \multicolumn{2}{|c|}{ Total population $(n=128)$} & \multicolumn{2}{|c|}{ Choanal atresia (right) $(n=32)$} & \multicolumn{2}{|c|}{ Control $(n=96)$} \\
\hline & Mean & Sd & Mean & Sd & Mean & Sd \\
\hline Age at CT (days) & 451.4 & 647.9 & 439.1 & 673.1 & 455.5 & 639.5 \\
\hline Maxillary length (mm) & 34.9 & 5.2 & 32.7 & 4.6 & 35.7 & 5.1 \\
\hline Right piriform aperture width (mm) & 10.3 & 1.9 & 10.0 & 2.1 & 10.4 & 1.8 \\
\hline Left piriform aperture width (mm) & 10.4 & 2.1 & 10.7 & 2.9 & 10.3 & 1.7 \\
\hline Left choanal width $(\mathrm{mm})$ & 8.2 & 1.7 & 8.3 & 2.5 & 8.2 & 1.4 \\
\hline Right choanal width (mm) & 7.3 & 1.6 & 6.2 & 1.5 & 7.6 & 1.5 \\
\hline
\end{tabular}

Table 3

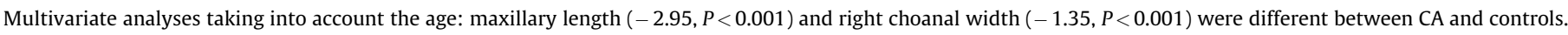

\begin{tabular}{|c|c|c|c|c|c|}
\hline & & Estimate & Sd & $\mathrm{T}$ & $P$ \\
\hline \multirow[t]{2}{*}{ Maxillary length } & Atresia & -2.95 & 0.55 & -5.33 & $<0.001$ \\
\hline & Age & 0.006 & 0.0003 & 17.55 & $<0.001$ \\
\hline \multirow[t]{2}{*}{ Right piriform aperture width } & Atresia & -0.32 & 0.26 & -1.23 & 0.22 \\
\hline & Age & 0.002 & 0.0002 & 12.2 & $<0.001$ \\
\hline \multirow[t]{2}{*}{ Left piriform aperture width } & Atresia & 0.43 & 0.35 & 1.24 & 0.22 \\
\hline & Age & 0.002 & 0.0002 & 8.13 & $<0.001$ \\
\hline \multirow[t]{2}{*}{ Left choanal width } & Atresia & 0.19 & 0.27 & 0.69 & 0.49 \\
\hline & Age & 0.002 & 0.0002 & 8.79 & $<0.001$ \\
\hline \multirow[t]{2}{*}{ Right choanal width } & Atresia & -1.35 & 0.25 & -5.32 & $<0.001$ \\
\hline & Age & 0.001 & 0.0002 & 7.36 & $<0.001$ \\
\hline
\end{tabular}

24-36 months of age and 6/32 were over 36 months of age. 20/32 (62.5\%) had right CA, $12 / 32$ had left CA; $18 / 32$ (56.25\%) had combined forms, $12 / 32$ (37.5\%) had bony CA and 6/32 (6.25\%) had membranous CA. Ninety-six control CT-scans were also included. CA and control groups were similar in terms of age: mean $439.1 \pm 673.1$ days in CA group, $455.5 \pm 639.5$ days in control group $(P>0.05)$. The sex-ratio in the control group was 0.5 .
The CCC measured for each landmark and the OCCC were all above 0.99 , indicating good intra- and inter-operator reproducibility, and thus reliable landmarking.

The Procrustes distance between the left and right CA groups was not significant $(\mathrm{d}=0.273, P=0.02)$. The Procrustes distances between 'left CA and controls', and 'right CA and controls' were both significant ( $d=0.352, P<0.0001 ; d=0.260, P<0.0001$ respectively). We thus
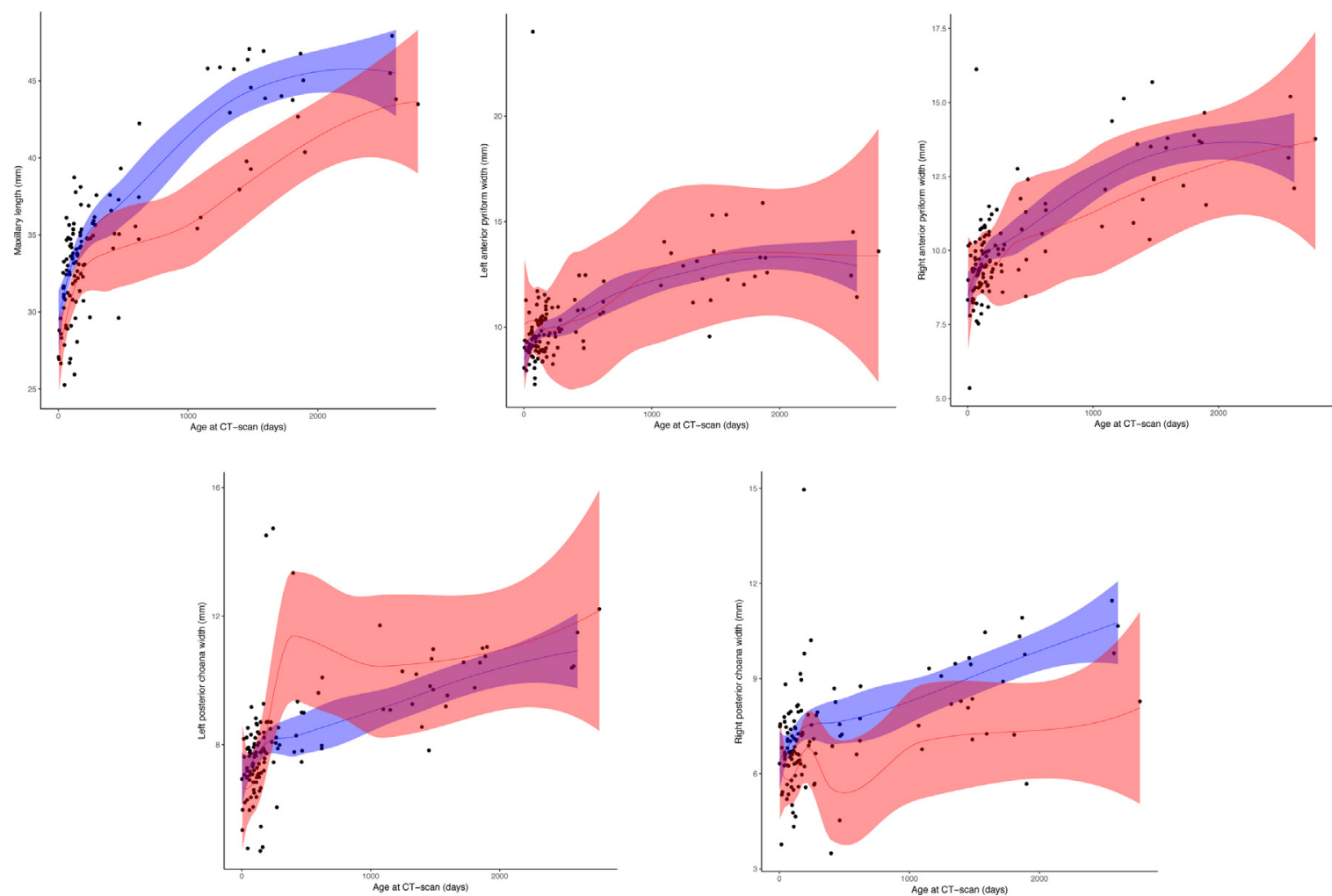

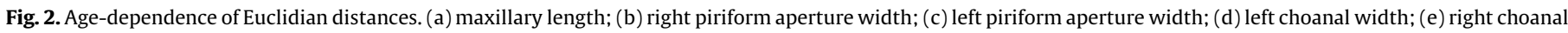
width. Dots: distribution for each patient. Red curves: choanal atresia group; blue curves: control group. 
Table 4

Growth dynamics were similar in atretric and control patients. $P$ : interaction test between slopes from control and atretic patients.

\begin{tabular}{ll}
\hline Maxillary length & 0.23 \\
Right piriform aperture width & 0.35 \\
Left piriform aperture width & 0.37 \\
Left choanal width & 0.14 \\
Right choanal width & 0.023 \\
\hline
\end{tabular}

clustered left and right CAs within the same group after transforming left CAs into right forms. The global shape difference between CA and control patients was significant $(\mathrm{d}=0.280, P<0.0001)$.

Multivariate analysis on selected Euclidian distances (Table 2), with age taken into account, showed that the mean maxillary length and mean right choanal width were statistically shorter in the CA group when compared to controls $(P<0.001)$. Mean left choanal width, mean right and left piriform apertures were similar in CA and controls (Table 3).

Logistic regressions were conducted in order to study the growth dynamics of each of the five distances in both groups (Fig. 2). Qualitatively, growth was different for the maxillary length and the right choanal width. Left choanal width, right and left piriform aperture widths were similar regarding ontogenetic trajectories in both groups. More precisely, right choanal width only (that is the affected side in all patients after mirroring left CA) showed lower growth rates (Table 4).

\section{Discussion}

Our sample had a wider age range ( $0-7$ years) and was larger (32 patients) than previous studies who had included 9 [19] and 11 [20] patients under 3 years of age $[19,20,25]$. In spite of a relatively less balanced sex ratio (10/22 vs. 1 ), our sample was similar to the literature data in terms of CA type (bony, membranous or combined) and right prevalence (63\%).

The nasal cavities in CA were significantly different from controls. More precisely, the maxillary length (or septal length) was significantly reduced in the CA group. Nevertheless, there was no other major shape difference between the nasal cavities in CA and controls, and specifically, no anterior collapse - the piriform aperture dimensions were similar in both groups. In the literature, studies based on 2D distance computations had shown increased bony septal width $[19,20,26]$ and diminished nasopharyngeal length [19].

We considered CA as a model for assessing the theories implicating fetal respiration in the formation of nasal cavities, and could not find support for the mechanical role of pre-natal fluid flux in this process [19]. Ontogenetic trajectories furthermore indicated that all distances in the CA group had dynamics similar to the control group except for the choanal width on the affected side, which had significantly slower growth rates. These results indicated that nasal cavity growth in CA was not de-correlated to the growth of the rest of the craniofacial skeleton. This dynamic result further supported the most probably limited involvement of fetal respiration in pre-natal maxillary growth.

Previous studies had described the speed of choanal aperture growth in normal patients through 2D CT-scans and demonstrated a linear correlation between age and choanal width: the mean choanal aperture $(\mathrm{mm})$ responded to the following equation: $(0.2 \pm 0.09) \times($ age $)+(6.78 \pm 0.67)(25)$, or: $4.85+(0.04) \times$ (age in weeks) [25]. Interestingly, our 3D measurements did not follow linear growth patterns, and further investigations focused on normal patients are required, with larger cohorts, in order to confirm the non-linearity of choanal aperture growth.

\section{Conclusion}

The atretic nasal cavity was significantly different from controls but was not collapsed. The maxillary length and the choanal width were decreased in atretic patients when compared to control cases. There were no significant differences in terms of piriform aperture width on the atretic side when compared to controls. Growth speeds of atretic and control patients were correlated, except for the choanal width on the affected side. Significant nasal cavity growth thus occurred despite absent or abnormal prenatal amniotic flux.

A similar controlled approach could be applied to assess NC growth after CA surgery, and to other types of NC malformations such as piriform aperture stenosis, in order to further investigate the potential role of fluid flux in maxillary growth.

\section{Funding}

This research did not receive any specific grant from funding agencies in the public, commercial, or not-for-profit sectors.

\section{Disclosure of interest}

The authors declare that they have no competing interest.

\section{References}

[1] Flake CG, Ferguson CF. Congenital choanal atresia in infants and children. Ann Otol Rhinol Laryngol 1964;73:458-73.

[2] Corrales CE, Koltai PJ. Choanal atresia: current concepts and controversies. Curr Opin Otolaryngol Head Neck Surg 2009;17:466-70.

[3] Kwong KM. Current Updates on Choanal Atresia. Front Pediatr 2015;3:52.

[4] Ramsden JD, Campisi P, Forte V. Choanal atresia and choanal stenosis. Otolaryngol Clin North Am 2009;42:339-52 [x].

5] Burrow TA, Saal HM, de Alarcon A, Martin LJ, Cotton RT, Hopkin RJ. Characterization of congenital anomalies in individuals with choanal atresia. Arch Otolaryngol Head Neck Surg 2009;135:543-7.

[6] Lesciotto KM, Heuzé Y, Jabs EW, Bernstein JM, Richtsmeier JT. Choanal atresia and craniosynostosis: development and disease. Plast Reconstr Surg 2018:141:156-68

[7] Hengerer AS, Brickman TM, Jeyakumar A. Choanal atresia: embryologic analysis and evolution of treatment, a 30-year experience. The Laryngoscope 2008:118:862-6.

[8] Hengerer AS, Strome M. Choanal atresia: a new embryologic theory and its influence on surgical management. The Laryngoscope 1982;92:913-21.

[9] Mugnier A. Embryologie et développement bucco-facial. Paris: Masson et Cie, Julien Prélat; 1964

[10] W.J. Hamilton, J.D. Boyd, H.W. Mossman. Alimentary and respiratory systems, pleural and peritoneal cavities. In: Human Embryology. Cambridge: W. Heffer and sons limited.

[11] Wolf D, Foulds N, Daya H. Antenatal carbimazole and choanal atresia: a new embryopathy. Arch Otolaryngol Head Neck Surg 2006;132:1009-11.

[12] Di Gianantonio E, Schaefer C, Mastroiacovo PP, Cournot MP, Benedicenti F, Reuvers M, et al. Adverse effects of prenatal methimazole exposure. Teratology 2001;64:262-6.

[13] Barbero P, Valdez R, Rodríguez H, Tiscornia C, Mansilla E, Allons A, et al. Choanal atresia associated with maternal hyperthyroidism treated with methimazole: a case-control study. Am J Med Genet A 2008;146A:2390-5.

[14] Dupé V, Matt N, Garnier J-M, Chambon P, Mark M, Ghyselinck NB. A newborn lethal defect due to inactivation of retinaldehyde dehydrogenase type 3 is prevented by maternal retinoic acid treatment. Proc Natl Acad Sci U S A 2003;100:14036-41.

[15] Minoux M, Rijli FM. Molecular mechanisms of cranial neural crest cell migration and patterning in craniofacial development. Dev Camb Engl 2010;137: 2605-21

[16] Sanlaville D, Verloes A. CHARGE syndrome: an update. Eur J Hum Genet EJHG 2007:15:389-99.

[17] Mooney MP, Siegel MI, Kimes KR, Todhunter J. Premaxillary development in normal and cleft lip and palate human fetuses using three-dimensional computer reconstruction. Cleft Palate Craniofac J 1991;28:49-53 [discussion 54].

[18] Talmant J-C, Talmant J-C, Lumineau J-P. A functional approach in the primary treatment of labial-alveolar-velopalatine clefts for a minimum of sequels. Rev Stomatol Chir Maxillofac 2007;108:255-63.

[19] Aslan S, Yilmazer C, Yildirim T, Akkuzu B, Yilmaz I. Comparison of nasal region dimensions in bilateral choanal atresia patients and normal controls: a computed tomographic analysis with clinical implications. Int J Pediatr Otorhinolaryngol 2009;73:329-35

[20] Slovis TL, Renfro B, Watts FB, Kuhns LR, Belenky W, Spoylar J. Choanal atresia: precise CT evaluation. Radiology 1985;155:345-8. 
[21] Keustermans W, Huysmans T, Danckaers F, Zarowski A, Schmelzer B, Sijbers J, et al. High quality statistical shape modelling of the human nasal cavity and applications. R Soc Open Sci 2018;5:181558.

[22] Lin LI. A concordance correlation coefficient to evaluate reproducibility. Biometrics 1989;45:255-68.

[23] Barnhart HX, Haber M, Song J. Overall concordance correlation coefficient for evaluating agreement among multiple observers. Biometrics 2002;58:1020-7.
[24] Klingenberg CP. MorphoJ: an integrated software package for geometric morphometrics. Mol Ecol Resour 2011:11:353-7.

[25] Corsten MJ, Bernard PA, Udjus K, Walker R. Nasal fossa dimensions in normal and nasally obstructed neonates and infants: preliminary study. Int J Pediatr Otorhinolaryngol 1996;36:23-30.

[26] Faust RA, Phillips CD. Assessment of congenital bony nasal obstruction by 3 dimensional CT volume rendering. Int J Pediatr Otorhinolaryngol 2001;61:71-5. 